\title{
Mycoplasma Pneumoniae Pneumonia: Walking Pneumonia Can Cripple the Susceptible
}

\author{
Bharat Bajantria ${ }^{\mathrm{a}}$, Omesh Toolsie ${ }^{\mathrm{a}, \mathrm{b}}$, Sindhaghatta Venkatram ${ }^{\mathrm{a}}$, \\ Gilda Diaz-Fuentes ${ }^{\text {a }}$
}

\begin{abstract}
Background: Mycoplasma pneumoniae pneumonia (MPP), or "walking pneumonia," is an atypical mild disease with varied clinical findings. Specifically, diagnosis is often controversial and understanding of disease presentation is limited. The goal of the study was to evaluate presentation, clinical associations and outcomes of MPP patients admitted to an inner-city hospital.
\end{abstract}

Methods: This was a retrospective analysis of adult patients diagnosed with MPP from January 2010 to January 2017. Primary outcomes were need for intensive care unit (ICU) care, ICU and hospital length of stay (LOS), presence of shock and need for mechanical ventilation (MV). Predictors of mortality were analyzed.

Results: Of the 203 patients analyzed, $16(8 \%)$ died. Relative to survivors, non-survivors were older $(65 \pm 21$ versus $53 \pm 18, \mathrm{P}=$ 0.009 ) and less frequently had obstructive airway disease (OAD; $\mathrm{P}$ $=0.003)$. Non-survivors also had significantly higher serum levels of lactic dehydrogenase (LDH), blood urea nitrogen (BUN), creatinine, transaminases and troponins. Finally, non-survivors more commonly exhibited shock, need for MV and bilateral lung infiltrates. There were no group differences in patient comorbidities, symptoms, antibiotic use or LOS. Predictors of mortality included age, OAD, low $\mathrm{CD}^{+} \mathrm{T}$-cell counts in human immunodeficiency virus-infected patients and elevated serum levels of $\mathrm{LDH}$, creatinine, BUN, leukocytes, transaminases and troponins.

Conclusion: Despite the availability of appropriate antibiotics, MPP incurs significant mortality and morbidity. Our study indicated that the recommended treatment includes prompt serological diagnosis, aggressive supportive care and presumptive antibiotics, especially in patients with poor prognosis.

Keywords: Atypical pneumonia; Mycoplasma pneumoniae; Walking pneumonia; Mycoplasma pneumoniae IgM titers

Manuscript submitted September 22, 2018, accepted October 16, 2018

aDivision of Pulmonary and Critical Care Medicine, Bronx Care Health System, Bronx, NY 10457, USA

${ }^{\mathrm{b}}$ Corresponding Author: Omesh Toolsie, Division of Pulmonary and Critical Care Medicine, Bronx Care Health System, Bronx, NY 10457, USA.

Email: otoolsie@bronxleb.org

doi: https://doi.org/10.14740/jocmr3592w

\section{Introduction}

Mycoplasma pneumoniae (MP) infections can commonly cause M. pneumoniae pneumonia (MPP), or "walking pneumonia." The prevalence of MPP is widely underestimated, largely because disease presentation is usually mild and MPP patients do not always seek medical attention. Furthermore, the lack of accurate diagnostic modalities for MPP makes diagnosis difficult and often controversial. Similar to MPP presentation, reports of mortality from MP infections show wide disparities. Among the elderly, mortality is reportedly around $8.0 \%[1,2]$, while young adults with no underlying disease are primarily affected by fulminant MPP, which accounts for $0.5-2 \%$ of all MPP cases [3]. Another study reported an in-hospital mortality of 30\% [4].

The objective of this study was to evaluate the clinical presentation, associations and outcomes of patients hospitalized for MPP in an inner-city hospital in New York City.

\section{Materials and Methods}

This study was approved by the hospital institutional review board (IRB), and the need for informed consent was waived (IRB number: 09081608).

\section{Study design}

This was a retrospective review of all adults admitted and diagnosed with MPP from January 2010 to January 2017 at Bronx Lebanon Hospital Center. Bronx Lebanon Hospital Center is the largest voluntary, not-for-profit health care system serving the South and Central Bronx, with 972 beds at two major hospital divisions. This hospital cares for a large, underserved and poor population with multiple comorbid conditions.

\section{Methods}

This study included all adult patients admitted to Bronx Lebanon Hospital Center from January 2010 to January 2017 with the diagnosis of pneumonia and serum MP IgM antibodies $\geq$ $770 \mathrm{U} / \mathrm{mL}$. Exclusion criteria included: 1) patients without pneumonia as the admitting diagnosis and 2) patients with serum MP IgM antibodies $<770 \mathrm{U} / \mathrm{mL}$. 
Table 1. Patient Demographic Characteristics and Comorbid Conditions

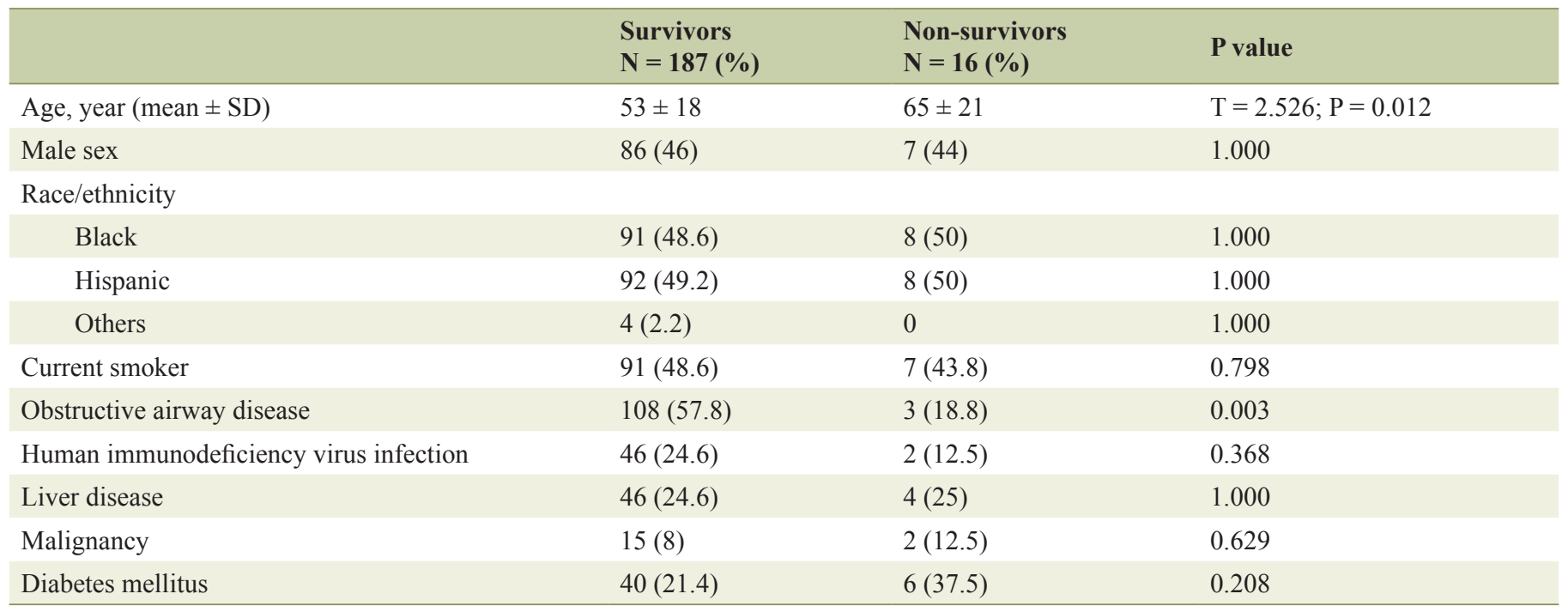

In previous MPP studies, diagnosis modality varies widely and includes cultures, polymerase chain reaction (PCR), serology and antigen detection techniques. Of these, serology has a sensitivity and specificity of approximately $90 \%$ for titers above $32 \mathrm{U} / \mathrm{mL}$. Thus, in our study, we used serology as the diagnosis modality with threshold serum MP IgM antibody titers of $770 \mathrm{U} / \mathrm{mL}$ to diagnose MPP.

For all patients, we recorded baseline general characteristics and presence of comorbid conditions such as chronic kidney disease, diabetes mellitus, obstructive airway disease (OAD), coagulopathy, malignancy, infection with human immunodeficiency virus (HIV) and liver disease. Data regarding laboratory parameters, chest imaging results and antibiotic management were also collected. Primary outcomes included admission to the intensive care unit (ICU), ICU and hospital length of stay (LOS), presence of septic shock and need for mechanical ventilation (MV). Predictors of inpatient mortality were also analyzed.

\section{Analysis}

In this study, we compared survivors with non-survivors. Group differences for continuous variables and proportions were analyzed with a Mann-Whitney $U$ test and Fisher's exact test, respectively. A binomial logistic regression model was constructed to test the predictive power of age, OAD, CreatinineMax, BUNMax, Infiltrates, MycAbLevel, ICULOSDays, Shock, MV and Macrolide use on patient survival. Association significance was measured with a two-tailed $t$-test. All tests were performed using Statistical Package for Social Sciences v.17 (SPSS Inc., Chicago, IL, USA), with a P value of $\leq 0.05$ considered significant.

\section{Results}

During the study period, we identified a total of 203 patients composed of 54\% women and with an average age of 53 years. Of these patients, $24(12 \%)$ were admitted from a skilled nursing facility and the rest came from home. The majority of patients $(92 \%)$ survived hospitalization. Survivors were significantly younger than non-survivors $(53 \pm 18$ versus $65 \pm 21, \mathrm{P}=0.012)$ and more frequently had OAD $(\mathrm{P}=0.003)$. In our cohort, there were also frequent comorbidities, particularly $\mathrm{OAD}$ and tobacco abuse, but there were no group differences in patient race, smoking, liver disease, malignancy, diabetes or HIV infection (Table 1). Of note, not all the patients had HIV test available.

There were also no group differences in symptoms at disease presentation. Indeed, $91 \%$ of patients presented with respiratory and gastrointestinal complaints. However, cough was more frequent among survivors. Furthermore, exacerbation of OAD was seen in $19.7 \%$ of patients, and all required systemic steroids (Table 2). For 32 (67\%) of the 48 HIV-infected patients, $\mathrm{CD}^{+} \mathrm{T}$-cell counts were available, which revealed that although there were no group differences in this measure, $30 \%$ of those patients had acquired immunodeficiency syndrome.

Analysis of different laboratory parameters revealed that non-survivors had significantly higher serum lactic dehydrogenase ( $\mathrm{LDH} ; 2,113 \pm 1,476$ versus $346 \pm 227, \mathrm{P}=0.0001$ ), blood urea nitrogen (BUN; $54 \pm 40$ versus $25 \pm 20, \mathrm{P}=0.0001$ ), creatinine $(3.3 \pm 3$ versus $1.3 \pm 1.0, \mathrm{P}=0.0001)$ and leukocyte counts $(\mathrm{P}=0.0001)$. Non-survivors also had significantly higher liver function tests and serum troponins (Table 3 ). Thus, these values may be predictive of patient prognosis.

Comparisons of chest imaging results also revealed group differences. In the initial chest roentgenogram, lung infiltrates were observed in only $80 \%$ of survivors but in $100 \%$ of nonsurvivors, and non-survivors more commonly had bilateral infiltrates (10 versus 57, $\mathrm{P}=0.0128$ ). Normal chest $\mathrm{X}$-rays (CXRs) were observed in $18 \%$ of patients, primarily in survivors. Relatively few patients (33\%) underwent a chest computerized tomography (CT) scan to evaluate respiratory symptoms, but all that did, had abnormal scans (Table 4). Thus, the results of chest imaging varied among MPP patients, including 
Table 2. Symptoms and Extrapulmonary Involvement of Patients With MPP

\begin{tabular}{|c|c|c|c|}
\hline $\operatorname{Symptom}(s)$ & $\begin{array}{l}\text { Survivors } \\
N=187(\%)\end{array}$ & $\begin{array}{l}\text { Non-survivors } \\
\mathrm{N}=16(\%)\end{array}$ & P value \\
\hline Chest pain & $23(12.3)$ & 0 & 0.23 \\
\hline Dyspnea & $126(67.3)$ & $8(50)$ & 0.17 \\
\hline Cough & $133(71.1)$ & $5(31.2)$ & 0.003 \\
\hline Gastrointestinal & $35(18.7)$ & $4(25)$ & 0.51 \\
\hline Fever & $100(53.4)$ & $10(62.5)$ & 0.60 \\
\hline Musculoskeletal & $2(1)$ & $3(18.7)$ & 0.003 \\
\hline Exacerbation of obstructive airway disease (OAD) & $39(20.8)$ & $1(6.2)$ & 0.204 \\
\hline Patients given systemic steroids for OAD & $39(20.8)$ & $1(6.2)$ & 0.204 \\
\hline \multicolumn{4}{|l|}{ Extrapulmonary involvement of MP } \\
\hline Cardiovascular & $39(20.8)$ & $11(68.8)$ & 0.0001 \\
\hline Hematological & $29(15.5)$ & $3(18.8)$ & 0.72 \\
\hline Musculoskeletal & $106(56.7)$ & $16(100)$ & 0.0003 \\
\hline Pulmonary involvement & $150(80.2)$ & $16(100)$ & 0.047 \\
\hline
\end{tabular}

some that had initially normal CXRs, but bilateral infiltrates were associated with non-survivors. Multi-organ involvement, primarily of the liver, lung, kidney or cardiovascular system, was common, particularly in non-survivors (Table 2).

We then compared antibiotic management strategies to determine if this predicted patient survival. Appropriate and timely (within $24 \mathrm{~h}$ of admission) antibiotics were received by $160(79 \%)$ patients, which corresponded to $82 \%$ of survivors and $38 \%$ of non-survivors (Table 5 ). This highlights the importance of antibiotic treatment to MPP patient survival.

Finally, we compared outcomes in survivors and non-survivors. Eighty (39\%) patients required ICU care, most of them in the non-survivor group. Non-survivors were more likely to experience shock (5 versus $13, \mathrm{P}=0.007)$ and require $\mathrm{MV}(11$ versus $16, \mathrm{P}=0.0001)$. However, there were no group differences in the ICU or hospital LOS (Table 6). Of the survivors, discharge dispositions were as follows: $22(12 \%)$ patients to a skilled nursing facility, $12(6 \%)$ to hospice, $4(2 \%)$ to a tertiary care center and the remaining $149(80 \%)$ to home.

\section{Discussion}

We first analyzed common MPP patient demographics and co-

Table 3. Laboratory Parameters

\begin{tabular}{llll}
\hline $\begin{array}{l}\text { Laboratory parameters } \\
\text { Mean } \pm \text { SD }(\mathbf{n})(\text { units) }\end{array}$ & $\begin{array}{l}\text { Survivors } \\
\mathbf{N}=\mathbf{1 8 7}\end{array}$ & $\begin{array}{l}\text { Non-survivors } \\
\mathbf{N}=\mathbf{1 6}\end{array}$ & P value \\
\hline Serum lactic dehydrogenase (unit/L) & $346 \pm 227(\mathrm{~N}=81)$ & $2,113 \pm 1,476(\mathrm{~N}=4)$ & $\mathrm{T}=9.627 ; \mathrm{P}=0.0001$ \\
Serum haptoglobin $(\mathrm{mg} / \mathrm{dL})$ & $169 \pm 126(\mathrm{~N}=29)$ & $132 \pm 120(\mathrm{~N}=3)$ & $\mathrm{T}=0.486 ; \mathrm{P}=0.631$ \\
Serum creatinine kinase (unit/L) & $558 \pm 1,645(\mathrm{~N}=106)$ & $1,029 \pm 2,780(\mathrm{~N}=16)$ & $\mathrm{T}=0.962 ; \mathrm{P}=0.338$ \\
Serum blood urea nitrogen & $25 \pm 20$ & $54 \pm 40$ & $\mathrm{~T}=5.032 ; \mathrm{P}=0.0001$ \\
Serum creatinine $(\mathrm{mg} / \mathrm{dL})$ & $1.3 \pm 1.0$ & $3.3 \pm 3$ & $\mathrm{~T}=6.076 ; \mathrm{P}=0.0001$ \\
White blood cells $(\mathrm{k} / \mu \mathrm{L})$ & $13.7 \pm 7.0$ & $22.7 \pm 9.1$ & $\mathrm{~T}=4.814 ; \mathrm{P}=0.0001$ \\
Platelets $(\mathrm{k} / \mu \mathrm{L})$ & $269 \pm 134$ & $241 \pm 126$ & $\mathrm{~T}=0.806 ; \mathrm{P}=0.421$ \\
Serum aspartate transaminase (unit/L) & $84 \pm 260(\mathrm{~N}=169)$ & $1,789 \pm 305(\mathrm{~N}=16)$ & $\mathrm{T}=7.179 ; \mathrm{P}=0.0001$ \\
Serum alkaline transaminase $(\mathrm{unit} / \mathrm{L})$ & $59 \pm 203(\mathrm{~N}=169)$ & $566 \pm 936(\mathrm{~N}=16)$ & $\mathrm{T}=5.853 ; \mathrm{P}=0.0001$ \\
Troponin T $(\mathrm{ng} / \mathrm{mL})$ & $1.0 \pm 0.3(\mathrm{~N}=39)$ & $0.45 \pm 0.64(\mathrm{~N}=11)$ & $\mathrm{T}=2.591 ; \mathrm{P}=0.013$ \\
Mycoplasma IgM titers $(\mathrm{U} / \mathrm{mL})$ & $1,555 \pm 1,127$ & $1,366 \pm 582$ & $\mathrm{~T}=0.662 ; \mathrm{P}=0.509$ \\
\hline
\end{tabular}


Table 4. Comparison of Chest Imaging Results

\begin{tabular}{llll}
\hline Chest X-ray (CXR) on admission & $\begin{array}{l}\text { Survivors } \\
\mathbf{N = 1 8 7}(\mathbf{\%})\end{array}$ & $\begin{array}{l}\text { Non-survivors } \\
\text { N=16 (\%) }\end{array}$ & P value \\
\hline CXR-unilateral infiltrates & $93(50 \%)$ & $6(37.5)$ & 0.438 \\
CXR-bilateral infiltrates & $57(30.5)$ & 0 & 0.0128 \\
CXR-normal & $37(19.8)$ & & 0.047 \\
Chest computed tomography (CT) scan on admission & & $3(18.7)$ & 0.27 \\
Number of patients with chest CT scan & $64(34.2)$ & $1(33.3)$ & 1.000 \\
Ground-glass opacification/airspace consolidation & $22(34.4)$ & $1(33.3)$ & 0.355 \\
Nodules/masses & $8(12.5)$ & 0 & 1.000 \\
Bronchovascular thickening/linear opacities & $13(6.9)$ & 0 & 1.000 \\
\hline Pleural effusion(s) & $15(23.4)$ & 0 & 1.000 \\
Lymphadenopathy & $4(6.3)$ & 0 & 1.000 \\
Bronchiectasis & $7(10.9)$ & 0 & 1.000 \\
Cavitary lesions & $2(3)$ & $1(33.3)$ & 0.08 \\
\hline Acute pulmonary embolism & $1(1.5)$ & 0 & 1.000 \\
\hline Normal CXR with abnormal chest CT & $8(12.5)$ & & \\
\hline
\end{tabular}

Table 5. Comparison of Antibiotic Management

\begin{tabular}{llll}
\hline Treatment characteristics & $\begin{array}{l}\text { Survivors } \\
\mathbf{N}=\mathbf{1 8 7}(\mathbf{\%})\end{array}$ & $\begin{array}{l}\text { Non-survivors } \\
\mathbf{N = 1 6}(\mathbf{\%})\end{array}$ & P value \\
\hline Macrolides & $124(66.3)$ & $3(18.8)$ & 0.0003 \\
Quinolones & $14(7.5)$ & $1(6.2)$ & 1.000 \\
Both: macrolides and quinolones & $16(8.5)$ & $2(12.5)$ & 0.639 \\
Either & $138(73.8)$ & $6(37.5)$ & 0.007 \\
No antibiotics to cover for mycoplasma & $33(17.6)$ & $10(62.5)$ & 0.0002 \\
Appropriate antibiotic & $154(82.3)$ & $6(37.5)$ & 0.0002 \\
Appropriate antibiotics after 24 h & $14(7.5)$ & $1(6.2)$ & 1.000 \\
Days of appropriate antibiotic & $5.82 \pm 3.45(\mathrm{~N}=154)$ & $6.33 \pm 5.47(\mathrm{~N}=6)$ & $\mathrm{T}=0.347 ; \mathrm{P}=0.729$ \\
\hline
\end{tabular}

morbidities. Our study supports the concept that among adults, illness severity increases in the elderly population $[1,2]$. For example, a prospective analysis of 64 MP patients older than 65 years showed that $9 \%$ of patients were admitted to the ICU, and one-third of these patients required MV [1]. Similarly, in our cohort, non-survivors were significantly older than survivors (Table 1). Previous reports also indicate that MPP is more common in patients with an underlying OAD, such as bronchial asthma or chronic obstructive pulmonary disease (COPD) [5-8]. In our study, 55\% of our cohort had history of OAD, supporting that OAD increases MPP incidence. Recent studies suggest a strong association between MP infections and exacerbation of asthma [9-12]. For example, asthmatics hospitalized for an exacerbation are six times more likely to have an MP infection [13], and refractory asthma is associated with the CARDS toxin assay [14]. Furthermore, PCR of

Table 6. Comparison of Outcomes Between Survivors and Non-Survivors

\begin{tabular}{llll}
\hline Outcomes & $\begin{array}{l}\text { Survivors } \\
\mathbf{N}=\mathbf{1 8 7}(\mathbf{\%})\end{array}$ & $\begin{array}{l}\text { Non-survivors } \\
\mathbf{N}=\mathbf{1 6}(\mathbf{\%})\end{array}$ & P value \\
\hline Intensive care unit (ICU) admission & $68(36.3)$ & $12(75)$ & 0.003 \\
Septic shock & $13(6.9)$ & $5(31.2)$ & 0.007 \\
Mechanical ventilation & $16(8.5)$ & $11(68.8)$ & 0.0001 \\
ICU length of stay (days) & $5.6 \pm 10$ & $6.0 \pm 8.2$ & $\mathrm{~T}=0.155 ; \mathrm{P}=0.877$ \\
Hospital length of stay (days) & $7.3 \pm 8.0$ & $9.4 \pm 10$ & $\mathrm{~T}=0.987 ; \mathrm{P}=0.325$ \\
\hline
\end{tabular}


upper airway samples revealed that $40 \%$ of controlled asthma adults had an MP infection, although none had MP antibodies. Together, these data suggest that allergic airway inflammation predisposes the host response to MP infections, implicating MP prevalence in chronic asthma and its role in inflammatory respiratory disease. Interestingly, Kraft et al showed that among asthmatics with MP infections, 6 weeks of clarithromycin treatment improved lung function [15]. In the context of COPD, Smith et al demonstrated that irrespective of COPD severity, the number of exacerbations was higher in patients with MP infections relative to un-infected individuals [5]. Finally, a retrospective study by Lieberman et al showed that MP was the only atypical infection associated with hospitalization for exacerbation of bronchial asthma or $\operatorname{COPD}[6,13]$. In total, these data coincide with our study results demonstrating that more than $50 \%$ of MP patients had concomitant OAD.

In terms of disease presentation, most of our patients presented with non-specific respiratory symptoms, which is consistent with the results from previous studies. Respiratory symptoms associated with MP infections ranged from minor issues such as productive or dry cough secondary to tracheobronchitis to more serious symptoms like exacerbation of OADs or respiratory failure. Other reported symptoms include lung abscess, bronchiectasis, organizing pneumonia, cellular bronchiolitis, pulmonary embolism, pleural effusion and empyema, chronic interstitial fibrosis, diffuse alveolar damage and adult respiratory distress syndrome [9, 16, 17].

Almost $90 \%$ of our patients also had some extrapulmonary finding, as demonstrated by symptoms or laboratory findings. This is unsurprising, as MP infections can affect the cardiovascular, digestive, neurological, hematological and dermatological systems [16-19]. Indeed, extrapulmonary involvement associated with MPP typically results from three mechanisms: direct, indirect or vascular occlusion triggered by infection. Fatal complications of MP infection include acute respiratory distress syndrome, disseminated intravascular coagulation, hemophagocytic syndrome, acute disseminated encephalomyelitis, thrombotic events and Stevens-Johnson syndrome [16].

In general, features of MPP in chest imaging are non-specific, although four of the most common patterns are peribronchial and perivascular interstitial infiltrates (49\%), airspace consolidation (38\%), reticulonodular opacification $(8 \%)$ and nodular or mass-like opacification $(5 \%)$. More uncommon findings are pleural effusion and hilar lymphadenopathy [20]. Interestingly, of the 64 patients in our study that underwent chest CTs, cavities were seen in two (3\%) patients and lung masses/nodules in seven $(10 \%)$. In our patients, the most common findings in chest CTs were airspace consolidation, ground-glass opacification and pleural effusions. These features correspond to those previously reported in the literature. For example, Reittner et al observed that among $28 \mathrm{MPP}$ patients undergoing high-resolution chest CTs, the most common patterns were airspace consolidation and ground-glass attenuation [20]. In the pediatric population, however, focal or bilateral reticulonodular opacification are most indicative of MPP [21]. Additionally, normal CXRs have been previously reported in less than 5\% of MPP cases [21]. By contrast, $18 \%$ of our patients presented with normal CXRs. We suspect that this discrepancy was due to earlier disease presentation in our study than in previous reports.

MP infections are primarily managed using antibiotics that act on the bacterial ribosome to either inhibit protein synthesis or DNA replication. Effective antibiotics against MP include macrolides, ketolides, streptogramins, tetracyclines and fluoroquinolones [9]. Our study suggests that appropriate and timely antibiotic administration could increase chance of survival; however, this conclusion is limited by the low number of non-survivors in our cohort and the presence of acute respiratory failure and shock among those patients. Interestingly, 10\% of our patients survived despite not receiving appropriate antibiotics. This is not entirely surprising, as previous reports suggest that some MP infections can spontaneously resolve within 7 - 10 days of disease manifestation [22]. In general, though, antibiotics seem to be important for MPP patient outcomes. Indeed, a prospective epidemiological study of 343 patients with community-acquired pneumonia showed that identification of the etiological microorganism did not affect the mortality rate, but delays in antibiotic treatment increased mortality [23]. Miyashita et al further emphasized the importance of early antibiotic administration and the positive outcomes associated with systemic corticosteroid treatment, as delayed administration of appropriate antibiotics exacerbated MPP severity [24]. Together, these findings highlight the importance of early and appropriate antibiotic administration.

Reports of mortality from MP infections vary widely, ranging from less than $5-30 \%[4,9,24,25]$. In the elderly specifically, mortality occurs in approximately $8.0 \%$ of cases, which is similar to our findings [24, 25]. Khoury et al reported that for patients with MP infections, there was an overall ICU mortality rate of $26.5 \%$ and a hospital mortality of $29.4 \%$ [4]. For patients over 65 years specifically, the ICU mortality rate was $38.8 \%$ relative to $18 \%$ in the 19 - to 64 -year-age group. By contrast, for our patients, ICU mortality was lower at 15 . Furthermore, in the Khoury et al.'s study, overall patient mortality correlated with higher APACHE II scores and multiple comorbidities. Extrapulmonary complications of MPP may also contribute to mortality, especially serious cardiovascular and thrombotic complications for which antibiotic therapy would have no effect. Smoking may also lead to fulminant MP and adverse outcomes [3].

There were few limitations to our study. First, this was a single-center, retrospective study that captured adults with MP infections severe enough to require hospitalization. Thus, conclusions from our study must be restricted to patients requiring hospitalization. Second, our description of extrapulmonary involvement was restricted to complications severe enough to be captured in the medical records. Third, we analyzed only short-term all-cause hospital mortality, and few of our patients required transfer to hospice or tertiary center, so it is possible that long-term mortality rates would be higher. Lastly, we did not exclude patients with co-infections of the lung.

Strengths of our study include having a large cohort in an underserved urban population with multiple comorbidities. Furthermore, in our study, diagnosis of MP infections was based mainly on serology. Most studies diagnosed MP infections using serology. Cultures are rarely used, while PCR is expensive and not readily available. PCR results also become negative sooner than serological ones once antibiotic therapy 
commences [9]. Alternatively, IgM assay sensitivity increases with the duration of symptoms, approaching more than $70 \%$ sensitivity and a positive predictive value close to $80 \%$ after 16 days of symptoms [26]. Interestingly, in the adult population, results from PCR and serology correlate well, and using both techniques together improves the reliability and accuracy of MPP diagnosis [27-29]. It is clear that in symptomatic adults, detection of MP would be considered an infection [9]. Hence, our study represents real-world diagnostic modalities for MP infection, making it more translatable to everyday medical practice.

In conclusion, MPP that requires hospitalization is associated with patient mortality and morbidity, particularly in patients with poor prognostic features. MPP patients requiring hospitalization are usually middle-aged with associated comorbidities such as OAD. However, accurate diagnostic tools and understanding of MPP presentation are still limited. In particular, the myriad of clinicoradiological presentations for this disease make diagnosis difficult. Recognition of the different imaging features, including normal radiological findings, cavities and nodules, is required for prompt and specific disease treatment. Our study revealed that such treatment should include an early, empirical antibiotic regimen including coverage for possible MPP, prompt serological diagnosis and careful evaluation for extrapulmonary involvement. Aggressive care is recommended, especially for elderly patients and those with poor prognostic features. Our study contradicts the belief that MPP is a "benign" disease, as $12 \%$ of our patients were disabled enough to require transfer to a skilled nursing facility. Ultimately, our study highlights the morbidity and mortality of patients suffering from MPP.

\section{Disclosure}

All authors have nothing to disclose.

\section{Author Contributions}

All authors were involved in design of the study as well as data acquisition, analysis and interpretation of data.

\section{Definitions}

Mechanical ventilation (MV): invasive mechanical ventilation; septic shock: a patient requiring vasopressor to maintain mean arterial pressure $>65 \mathrm{~mm} \mathrm{Hg}$ despite adequate fluid resuscitation; mortality: in-hospital all-cause mortality; CreatinineMax: highest serum creatinine recorded during admission; BUNMax: highest serum BUN recorded during admission; MycAbLevel: serum mycoplasma IgM antibody.

\section{Abbreviations}

MPP: Mycoplasma pneumoniae pneumonia; MP: Mycoplasma pneumoniae; HIV: human immunodeficiency virus; LDH: lactic dehydrogenase; BUN: blood urea nitrogen; ICU: intensive care unit; LOS: length of stay; MV: mechanical ventilation; OAD: obstructive airway disease; COPD: chronic obstructive pulmonary disease; CXR: chest X-ray; CT: computed tomography

\section{References}

1. Marrie TJ. Mycoplasma pneumoniae pneumonia requiring hospitalization, with emphasis on infection in the elderly. Arch Intern Med. 1993;153(4):488-494.

2. Niederman MS, Brito V. Pneumonia in the older patient. Clin Chest Med. 2007;28(4):751-771, vi.

3. Chan ED, Welsh CH. Fulminant Mycoplasma pneumoniae pneumonia. West J Med. 1995;162(2):133-142.

4. Khoury T, Sviri S, Rmeileh AA, Nubani A, Abutbul A, Hoss S, van Heerden PV, et al. Increased rates of intensive care unit admission in patients with Mycoplasma pneumoniae: a retrospective study. Clin Microbiol Infect. 2016;22(8):711-714.

5. Smith CB, Golden CA, Kanner RE, Renzetti AD, Jr. Association of viral and Mycoplasma pneumoniae infections with acute respiratory illness in patients with chronic obstructive pulmonary diseases. Am Rev Respir Dis. 1980;121(2):225-232.

6. Lieberman D, Lieberman D, Ben-Yaakov M, Shmarkov O, Gelfer Y, Varshavsky R, Ohana B, et al. Serological evidence of Mycoplasma pneumoniae infection in acute exacerbation of COPD. Diagn Microbiol Infect Dis. 2002;44(1):1-6.

7. Park SJ, Lee YC, Rhee YK, Lee HB. Seroprevalence of Mycoplasma pneumoniae and Chlamydia pneumoniae in stable asthma and chronic obstructive pulmonary disease. J Korean Med Sci. 2005;20(2):225-228.

8. Muro S, Tabara Y, Matsumoto H, Setoh K, Kawaguchi T, Takahashi M, Ito I, et al. Relationship among chlamydia and mycoplasma pneumoniae seropositivity, IKZF1 genotype and chronic obstructive pulmonary disease in a general Japanese population: The Nagahama Study. Medicine (Baltimore). 2016;95(15):e3371.

9. Waites KB, Xiao L, Liu Y, Balish MF, Atkinson TP. Mycoplasma pneumoniae from the Respiratory Tract and Beyond. Clin Microbiol Rev. 2017;30(3):747-809.

10. Atkinson TP, Duffy LB, Pendley D, Dai Y, Cassell GH. Deficient immune response to Mycoplasma pneumoniae in childhood asthma. Allergy Asthma Proc. 2009;30(2):158-165.

11. Smith-Norowitz TA, Silverberg JI, Kusonruksa M, Weaver D, Ginsburg D, Norowitz KB, Durkin HG, et al. Asthmatic children have increased specific anti-Mycoplasma pneumoniae $\operatorname{IgM}$ but not $\operatorname{IgG}$ or $\operatorname{IgE}$-values independent of history of respiratory tract infection. Pediatr Infect Dis J. 2013;32(6):599-603.

12. Medina JL, Coalson JJ, Brooks EG, Winter VT, Chaparro A, Principe MF, Kannan TR, et al. Mycoplasma pneumoniae CARDS toxin induces pulmonary eosinophilic and lymphocytic inflammation. Am J Respir Cell Mol Biol. 
2012;46(6):815-822.

13. Lieberman D, Lieberman D, Printz S, Ben-Yaakov M, Lazarovich Z, Ohana B, Friedman MG, et al. Atypical pathogen infection in adults with acute exacerbation of bronchial asthma. Am J Respir Crit Care Med. 2003;167(3):406-410.

14. Peters J, Singh H, Brooks EG, Diaz J, Kannan TR, Coalson JJ, Baseman JG, et al. Persistence of communityacquired respiratory distress syndrome toxin-producing Mycoplasma pneumoniae in refractory asthma. Chest. 2011;140(2):401-407.

15. Kraft M, Cassell GH, Pak J, Martin RJ. Mycoplasma pneumoniae and Chlamydia pneumoniae in asthma: effect of clarithromycin. Chest. 2002;121(6):1782-1788.

16. Narita M. Classification of Extrapulmonary Manifestations Due to Mycoplasma pneumoniae Infection on the Basis of Possible Pathogenesis. Front Microbiol. 2016;7:23.

17. Mansel JK, Rosenow EC, 3rd, Smith TF, Martin JW, Jr. Mycoplasma pneumoniae pneumonia. Chest. 1989;95(3):639-646.

18. Narita M. Pathogenesis of extrapulmonary manifestations of Mycoplasma pneumoniae infection with special reference to pneumonia. J Infect Chemother. 2010;16(3):162169.

19. Mishra R, Cano E, Venkatram S, Diaz-Fuentes G. An interesting case of mycoplasma pneumonia associated multisystem involvement and diffuse alveolar hemorrhage. Respir Med Case Rep. 2017;21:78-81.

20. Reittner P, Muller NL, Heyneman L, Johkoh T, Park JS, Lee KS, Honda O, et al. Mycoplasma pneumoniae pneumonia: radiographic and high-resolution $\mathrm{CT}$ features in 28 patients. AJR Am J Roentgenol. 2000;174(1):37-41.

21. John SD, Ramanathan J, Swischuk LE. Spectrum of clinical and radiographic findings in pediatric mycoplasma pneumonia. Radiographics. 2001;21(1):121-131.
22. File TM, Jr., Segreti J, Dunbar L, Player R, Kohler R, Williams RR, Kojak C, et al. A multicenter, randomized study comparing the efficacy and safety of intravenous and/or oral levofloxacin versus ceftriaxone and/or cefuroxime axetil in treatment of adults with communityacquired pneumonia. Antimicrob Agents Chemother. 1997;41(9):1965-1972.

23. Luna CM, Famiglietti A, Absi R, Videla AJ, Nogueira FJ, Fuenzalida AD, Gene RJ. Community-acquired pneumonia: etiology, epidemiology, and outcome at a teaching hospital in Argentina. Chest. 2000;118(5):1344-1354.

24. Miyashita N, Obase Y, Ouchi K, Kawasaki K, Kawai Y, Kobashi Y, Oka M. Clinical features of severe Mycoplasma pneumoniae pneumonia in adults admitted to an intensive care unit. J Med Microbiol. 2007;56(Pt 12):16251629.

25. Miyashita N, Ouchi K, Kawasaki K, Oda K, Kawai Y, Shimizu H, Kobashi Y, et al. Mycoplasma pneumoniae pneumonia in the elderly. Med Sci Monit. 2008;14(8):CR387-391.

26. Beersma MF, Dirven K, van Dam AP, Templeton KE, Claas EC, Goossens H. Evaluation of 12 commercial tests and the complement fixation test for Mycoplasma pneumoniae-specific immunoglobulin $\mathrm{G}(\mathrm{IgG})$ and $\mathrm{IgM}$ antibodies, with PCR used as the "gold standard". J Clin Microbiol. 2005;43(5):2277-2285.

27. Parrott GL, Kinjo T, Fujita J. A Compendium for Mycoplasma pneumoniae. Front Microbiol. 2016;7:513.

28. He XY, Wang XB, Zhang R, Yuan ZJ, Tan JJ, Peng B, Huang $Y$, et al. Investigation of Mycoplasma pneumoniae infection in pediatric population from 12,025 cases with respiratory infection. Diagn Microbiol Infect Dis. 2013;75(1):22-27.

29. Loens K, Ieven M. Mycoplasma pneumoniae: current knowledge on nucleic acid amplification techniques and serological diagnostics. Front Microbiol. 2016;7:448. 